\title{
La institucionalización de la individualización del trabajo: el concepto de flexiguridad
}

\author{
Francisco J. TOVAR MARTÍNEZ \\ Profesor del Departamento de Sociología y Trabajo Social \\ Universidad de Valladolid \\ fjtovar@cps.ucm.es \\ fjtovar@soc.uva.es \\ Juan Carlos REVILLA CASTRO \\ Profesor del Departamento de Psicología Social \\ Universidad Complutense de Madrid \\ jcrevilla@cps.ucm.es
}

Recibido: 29-04-2010

Aceptado: 28-02-2011

\begin{abstract}
RESUMEN
El concepto de flexiguridad se está convirtiendo en un elemento central en las políticas europeas de empleo. En este artículo se rastrea el concepto desde sus orígenes hasta el día de hoy a partir de un análisis de discurso del documento monográfico elaborado por la Comisión Europea, en diálogo con la literatura sobre la materia. Como resultado, se muestra la carga no equilibrada que del concepto de flexiguridad realiza la Comisión. Del mismo modo, se muestra cómo dicho concepto es un buen ejemplo de la tendencia generalizada hacia la individualización de las políticas de empleo europeas y de los discursos gerenciales más recientes, descargando sobre el individuo toda la responsabilidad de su devenir laboral.
\end{abstract}

Palabras clave: flexibilidad, seguridad, políticas de empleo, Unión Europea, análisis del discurso

\section{The institutionalization of the individualization of work: the concept of flexicurity}

\begin{abstract}
The concept of flexicurity is becoming a central element in European employment policies. In this work the origins of the concept are traced through a discourse analysis of the monographic document elaborated by the European Commision on the subject in dialog with the literature on the subject. The unbalanced use of flexicurity as used by the Commision is shown. In the same vein, it will be analysed how this concept is a good example of the generalized tendency to the individualisation of European employment policies, in line with recent managerial discourses, which place on the subject the whole responsibility for their laboral present and future.
\end{abstract}

Key words: flexibility, labor security, employment policies, European Union, discourse analysis 
Francisco J. Tovar y Juan Carlos Revilla La institucionalización de la individualización del trabajo...

\section{REFERENCIA NORMALIZADA}

Tovar Martínez, F. J., y Revilla Castro, J. C., (2012). La institucionalización de la individualización del trabajo: el concepto de flexiguridad. Cuadernos de Relaciones Laborales Vol. 30, núm. p. 235-258.

SUMARIO: Introduction. 1. En busca de una política laboral común. 1.1 El MSE como proyecto político. 1.2. La Estrategia Europea de Empleo (EEE): de la empleabilidad a la flexiguridad. 2. La popularización de la flexiguridad. 3. La institucionalización de la individualización: la flexiguridad. 4. Conclusiones. Bibliografía.

\section{Introducción}

La fragilización de las relaciones laborales viene siendo en los últimos años un hecho demostrable en las estadísticas nacionales y supranacionales europeas que muestran la disminución de los contratos indefinidos o la pérdida de poder de los sindicatos, entre otras muchas evidencias (Vielle \& Walthery 2003). Dicha fragilización se ha traducido en una individualización de las relaciones laborales, esto es, un individuo que se convierte en el único responsable de su vida laboral, un individuo que negocia directamente sus condiciones con la empresa ya que lo que se busca es la negociación directa e individual entre jefe y empleado. En ese sentido, la individualización tendría su última expresión en las nuevas formas de sujeción del individuo en el lugar de trabajo. Esto es lo que, en esa misma dirección, se viene denominando como la psicologización política del trabajo (Crespo et al. 2005). Siguiendo los mismos parámetros teóricos, autores como Bauman (2000 y 2003), Alonso (1999, 2001 y 2007), Rifkin (1997) o Sennett (2006) vienen señalando una serie de características comunes de una nueva etapa que podríamos denominar capitalismo tardío. Profundizando en los elementos clave de esta nueva etapa, destaca el aumento de las desigualdades en la relación entre empleador y empleado donde la precariedad y la fragmentacion, entre otros, se han vuelto en elementos incontestables de esta nueva realidad.

Por otra parte, la Unión Europea (UE), en su pretensión de crear un modelo económico y social común donde se combinen un crecimiento económico sostenible con la mejora de las condiciones de vida y de trabajo a través del pleno empleo, trabajos de calidad, igualdad de oportunidades, protección social universal e implicación de los ciudadanos en la toma de decisiones, ha venido desarrollando una serie de políticas comunes de empleo creando diferentes vías para ponerlas en marcha. Dos de los momentos más importantes en este proceso fueron las cumbres de Ámsterdam y de Lisboa de 1997 y 2000, respectivamente. En ellas se fijaron un número de ambiciosos objetivos a conseguir en diez años que hicieran de la economía de la UE la más competitiva y dinámica del mundo, siendo capaz de compaginar el crecimiento económico con la mejora cuantitativa y cualitativa del empleo, y todo ello acompañado de una fuerte cohesión social (Commis. Europeenne, 2003). En este contexto nace el concepto de flexiguridad y se desarrolla a nivel europeo. Así, la flexiguridad se ha convertido en la protagonista en numerosos documentos de la UE, al igual que de muchos científicos sociales en busca de definiciones, explicaciones y desarrollos hacia la consecución de los objetivos de los citados 
encuentros (Ámsterdam 1997 y Lisboa 2000). Flexiguridad se entiende, a nivel institucional, como una determinada política de empleo encaminada a proporcionar flexibilidad y seguridad en el mercado de trabajo. A grandes rasgos, la flexibilidad se traduciría en la proliferación de tipos de contratos y, consecuentemente, en la multiplicación de situaciones por las cuales un trabajador puede ser despedido. Seguridad, por el contrario, se refiere al garante que permite al individuo transitar de una situación de actividad a otra de inactividad y viceversa, es decir, el suministro de herramientas para que permanezca activo y empleable.

Lo que pretendemos evidenciar en el presente artículo es la carga no equilibrada (Keune \& Jepsen 2007) de flexibilidad y seguridad del concepto de flexiguridad. A través del análisis de los principales de la Comisión Europea, se mostrará cómo la vertiente de la flexibilidad aparece con mayor precisión y concreción que la vertiente de la seguridad. Del mismo modo, nos adentraremos en el trabajo de transformación que la Comisión está realizando con respecto a lo que se entiende por seguridad, es decir, pasando de una seguridad que era entendida como la certeza del mantenimiento del trabajo a ser entendida como un garante de transiciones en la vida del individuo. Dichas observaciones tienen mucho que ver con la tendencia generalizada hacia la individualización-psicologización que en la última década se ha instaurado en el discurso gerencial, el cual ha colocado las características más personales del individuo en el centro del trabajo. Gestiones basadas en las competencias, el compromiso o el talento atestiguan una centralidad del trabajadorindividuo en detrimento del trabajador-colectivo, valorando de forma exclusiva las características y desempeños individuales.

El artículo se estructurará en dos grandes partes. Una primera de carácter introductorio donde se situará el concepto de flexiguridad dentro de la Estrategia Europea de Empleo (EEE) que, al mismo tiempo, está enmarcada dentro del denominado Modelo Social Europeo (MSE). Para ello, dedicaremos las primeras páginas a posicionarnos dentro del debate sobre la forma y el contenido que ha adquirido el MSE así como en las dos características fundamentales de la EEE: el concepto de activación, piedra angular del modelo en la última década del siglo XX que se definiría, a grandes rasgos, en una disponibilidad absoluta del individuo con las exigencias que tenga en cada momento el mercado y, en segundo lugar, el concepto de flexiguridad.

La segunda parte versará sobre nuestra hipótesis acerca de la institucionalización de la individualización en el trabajo a través del concepto de flexiguridad. Para ellos nos parece pertinente conectar dicho debate con el que desde hace años se está produciendo en la Sociología y Psicología Social del Trabajo. Profundizar en lo que se ha denominado "doctrinas psicologizantes" (Crespo et al. 2005) nos ayudará a contextualizar y encontrar paralelismos a lo que, desde un plano más institucional, se está produciendo con el concepto de flexiguridad. 
Francisco J. Tovar y Juan Carlos Revilla La institucionalización de la individualización del trabajo...

\section{En busca de una política laboral común}

El MSE ha sido centro de numerosas reflexiones acerca de su origen y significado. Explicar y formar parte del debate nos parece condición indispensable para contextualizar al máximo nuestro punto de vista sobre el concepto de flexiguridad.

\subsection{EI MSE como proyecto político}

El MSE se define como un proyecto de sociedad donde se combina el crecimiento sostenible con la mejora de las condiciones de trabajo y de vida. Para ello elementos como el pleno empleo, la calidad del empleo, la igualdad de oportunidad, la protección social universal y la inclusión social son cruciales para la consecución de dicho modelo (ETUC 2005). La primera definición de corte institucional que aparece sobre el MSE emerge a mediados de los años 90, en ella se habla de un conjunto de valores comunes, libertad personal, dialogo social, igualdad de oportunidades para todos y la adecuación de la seguridad social y la solidaridad para aquellos segmentos de la población débiles (European Commission, 1994). Durante todo este tiempo, las instituciones europeas se han encargado de producir numerosos discursos asentados en tres elementos (Serrano y Crespo, 2002):

- $\quad$ la naturalización histórica: La creación de un discurso que aparece como natural en el que se exponen todos los principios que las instituciones europeas pretenden dar por sentado.

- $\quad$ la dicotomía existente entre el panorama socioeconómico americano y europeo: La constante búsqueda de presentar los modelos americano y europeo como enfrentados y diferentes.

- $\quad$ el nuevo modo de articulación del vínculo social: El surgimiento de un nuevo tipo de ciudadanía vinculado al empleo tal y como lo entiende el MSE, es decir, sólo aquellos individuos activados o empleables (que pueden ser contratados porque están "disponibles" en términos de cualificación) pueden adquirir el estatus de ciudadanía y por ende poseer los derechos y las obligaciones que a tal condición se le suponen (Ocaña, J.C., 2000)

A pesar de las numerosas evidencias que muestran que el acento de la Unión Europea está en una gran mayor medida en el lado económico que en el social (Jepsen $\&$ Serrano 2006, Maarten, 2007), ¿por qué el modelo sobre el que se sustenta y por el que ha de avanzar la UE es denominado el Modelo Social Europeo? Esta denominación se entiende como un intento de creación de un modelo común mucho más aceptable, y consecuentemente legitimado, por la sociedad donde, paradójicamente, el énfasis real no coincidiría con el énfasis nominal del mismo, es decir, la diferencia entre el dicho y el hecho. Esta diferencia entre significado y significante, la podemos entender como la lucha en el terreno del lenguaje por la creación de un nuevo status quo (Fairclough, 2000) que, como se verá, también parece existir con 
el concepto de flexiguridad pues lo que sugiere nominalmente, no se corresponde con lo que hace referencia realmente.

Según Jepsen y Serrano (2006) existirían tres modos de entender el MSE. Un primer modo definido por la existencia de ciertos elementos comunes (instituciones y valores, entre otros) que son compartidos por los diferentes países europeos, los cuales implican una aproximación normativa; un segundo modo entendería el MSE como una serie de diferentes prototipos ideales, fundamentados a partir de diferentes modelos nacionales y, de los cuales, algunos serían los buenos ejemplos; y un tercero visto como un proyecto europeo que serviría tanto para enfrentarse a las cambiantes condiciones económicas como para favorecer la cohesión de los países miembros. Es en este tercer punto donde, al igual que las autoras, se sitúa nuestra visión del MSE, pues existen numerosas manifestaciones que señalan la pretensión de la UE de crear una serie de elementos comunes a partir del conjunto de países que conforman Europa, los cuales, a priori, no tendrían tantos elementos en común como afirma la primera interpretación. Del mismo modo, y como parece derivarse de las conclusiones de la cumbre de Lisboa, aunque existen diferentes realidades que sirven de ejemplo al conjunto de la UE, el surgimiento de nuevos conceptos y la vaguedad en la definición de los mismos parecen cabalgar en una misma dirección: la creación de una gran identidad europea que englobaría las distintas singularidades existentes, lo que se denomina como el proyecto político del MSE (Jepsen y Serrano, 2006).

Para entender el MSE como proyecto político es necesario contextualizarlo de una manera amplia con los cambios y las transformaciones que a inicios de los noventa influyeron tanto a Europa como al resto del mundo. El proceso de globalización ha tenido consecuencias en dos principales dimensiones: la económica y la social. La primera se caracteriza por la innovación tecnológica, la competitividad creciente y la globalización de todas las esferas (Castells, 2001). Sin sustituir a las anteriores, las nuevas formas de producción se han centrado en el desarrollo exacerbado de la tecnología y la comunicación, las cuales han tenido como principal consecuencia la superación de los tiempos de producción previos. Paralelamente diversos análisis sobre productividad han venido a demostrar que son los individuos de la empresa los que realmente confieren distinción a la misma. El cambio permanente ha provocado que la organización haya pasado de ser estática a ser dinámica y flexible, integrando y valorando el trabajo de los empleados que, en última instancia, son el recurso más valioso de la empresa. El empleado es el único recurso capaz de elevar su valor mientras los demás se deprecian con el tiempo, el único recurso capaz de manejar los demás recursos de la empresa (Mertens, 1996).

Por dimensión social nos referimos a todas aquellas transformaciones en el plano laboral que han tenido como objetivo a los trabajadores, sus derechos y sus obligaciones. Así pues, la principal transformación producida en el terreno laboral está muy en relación con la dimensión económica. El altísimo desarrollo tecnológico trajo consigo la desaparición de numerosas tareas manuales. También, la importancia concedida a lo que el individuo aporta a la organización ha provocado un gran cambio en los métodos para dirigir la empresa, donde la rápida sustitución y la 
búsqueda constante de las mejores habilidades ha dejado caduca la base que definía institucionalmente el contrato de trabajo del individuo: las biografías laborales a través de contratos asalariados a tiempo completo indefinidos y estandarizados, el control disciplinario por parte del empleador, prestaciones sociales, etcétera. Se ha instaurado una nueva norma social donde ni siquiera el mejor y más competente empleado está a salvo de formas mucho más autónomas, parasubordinadas y cambiantes (Alonso, 2007). Esta nueva norma social derrumba la propiedad social en que se fundamentaban el mundo del trabajo y la ciudadanía laboral, para pasar a la "propiedad del sí mismo", donde el individuo tiene que hacerse valer frente al resto a través de sus capacidades, competencias y capitales sociales y simbólicos (Castel y Haroche, 2001; Alonso, 2007).

Sería incorrecto no mencionar en este punto la labor de los Estados de bienestar europeos en el refortalecimiento o no de los cambios producidos en la globalización económica y social. A la vista de los hechos, podemos decir que dichos Estados, junto a las instituciones europeas, han sido unos excelentes transmisores del ideario de la nueva economía del siglo XXI a la sociedad. Desde el punto de vista del mercado de empleo, los Estados, históricamente, se han caracterizado por la búsqueda del pleno empleo sin renunciar a la cohesión social, pero, desde finales de los noventa hasta la actualidad, se han concentrado en buscar un aumento del número de empleos desarrollando formas flexibles de trabajo con la esperanza de facilitar la reducción del desempleo, proporcionando a los empleadores unas regulaciones laborales acordes con el nuevo contexto de máxima competitividad y cambio constante, regulaciones donde tanto el contrato como el despido se conviertan en instrumentos al servicio del mercado (Vielle \& Walthery, 2003). Es decir, una cohesión social que se complementa con lo comentado anteriormente en relación a la nueva norma social. Esta nueva cohesión social consistiría más en igualar a toda la mano de obra a través de unas características similares (empleables, competentes, activos y emprendedores), que en proporcionarles un sistema de protección robusto frente a las adversidades sociales y/o económicas.

\subsection{La Estrategia Europea de Empleo (EEE): de la empleabilidad a la flexiguridad}

La EEE sería la línea a seguir en materia de empleo por el MSE. Dicha estrategia está estrechamente ligada al White Paper presentado por la Comisión Europea en 1993. Bajo la dirección del, por entonces, presidente Jacques Delors, la Comisión Europea concedió una importancia notable al capítulo de empleo. Dicho trabajo fue continuado y profundizado en la cumbre de Luxemburgo (1997), en el tratado de Ámsterdam (1998) y, más ampliamente, en el tratado de Lisboa (2000).

El objetivo prioritario de la EEE es la disminución del nivel de desempleo a través del fomento de la empleabilidad, es decir, fomentar que todos los trabajadores potencialmente activos estén en condición de ser contratados gracias a su continua formación. En ese sentido habría que hacer mención a la calidad de los trabajos creados, pues la importancia de enfatizar soluciones rápidas al desempleo no debiera justificar la creación de empleos a cualquier coste (Goetschy, 1999). Poniendo 
toda la atención en la reducción cuantitativa del desempleo, han aparecido numerosas formas de empleo que no responderían con los objetivos de protección e integración social universal en los que se basa el MSE.

Los dos elementos clave de la empleabilidad son las políticas de activación y el concepto de flexiguridad. Ambos son complementarios entre sí, es decir, el uno cobra sentido con el otro y viceversa. Tanto el uno como el otro intervienen en la construcción de una nueva relación laboral donde el Estado de bienestar perdería gran parte de su protagonismo (protección social) en detrimento del individuo que se convierte en el gran protagonista de dichas políticas, transformando muchos de sus derechos en responsabilidades a las que responder para mantener o adquirir el estatus de ciudadanía, definida en sus orígenes como un sistema de garantías que asegurase a cada individuo un trato jurídico, a través de derechos sociales, como miembro pleno de una sociedad de iguales (Marshall, 1997). La nueva ciudadanía poco tiene que ver con aquella definida y otorgada después de la segunda Guerra Mundial y que fue adquiriendo mayor peso y significado en las siguientes décadas. En la nueva idea de ciudadanía todo lo que concierne a la protección (centralidad, sustentación y seguridad en el empleo) se considera disfuncional y obstaculizador para el modelo de activación (Alonso, 2007). En esa nueva construcción de las relaciones laborales es el propio mercado el que se convierte en el único elemento no problemático (Serrano, 2007), a su vez el trabajador se convierte en el responsable, para bien o para mal, de su vida laboral y el Estado retrocede en su papel de intentar adecuar el funcionamiento del mercado. El Estado ha de dinamizar al mercado sin interferir en él, para ello ha de colaborar a través del fomento de una mano de obra apropiada a la demanda empresarial: un trabajador que sea flexible, a la vez que activo y empleable, entre otras características. En esta nueva formulación de la cuestión social, los individuos y el Estado ocupan nuevas posiciones: por un lado el individuo autorregulado, responsable y protagonista de su devenir laboral (Crespo, Revilla y Serrano, 2005), tanto para lo bueno como para lo malo y, por el otro, el Estado retrocediendo en su rol proteccionista y garantista para pasar a ser mero avalista de las transiciones de aquellos, es decir ha pasado de ser un Estado del bienestar a un Estado de la productividad (Alonso, 2007) en busca del máximo rendimiento mercantil. Las leyes del mercado, por el contrario, siguen perteneciendo al mundo de lo incuestionable y lo natural (Alonso, 1999) con lo que en ningún momento son planteadas como problemáticas $\mathrm{y}$, consecuentemente, revisadas o transformadas. Su curso, el de las leyes, es un curso que se nos presenta como natural e inexorable.

Las políticas de activación pueden ser definidas como unas dinámicas explícitas introducidas en las políticas públicas que conllevan un nuevo diseño de ayudas, asistencias y políticas de protección social en términos de eficiencia e igualdad, al mismo tiempo que se mejoran las distintas funciones sociales del trabajo remunerado y de la participación en la fuerza de trabajo (Barbier, 2004) . Nacen como respuesta al gran número de inactivos que están dentro del mercado laboral pero que se encuentran en situación de desempleo. Esto no quiere decir que dichas políticas incidan en el mercado de trabajo a fin de favorecer la inserción profesional de los 
desempleados. La activación pretende producir individuos e identidades más ajustadas a las nuevas reglas del modelo productivo, esto es, plantea, como se ha comentado en el punto anterior, una nueva idea de ciudadanía.

Serrano (2007) realiza un acertado análisis de la forma de intervención característica de las políticas de activación, las cuales tienen tres rasgos fundamentales: el énfasis en la contractualización, la centralidad del empleo y la perspectiva individualizadora.

Con respecto la primera, el contrato social pasaría a ser una metáfora central en el asentamiento de las políticas de activación. La autora pone de relieve el carácter moral de dicho contrato. Esta mutación provoca que los individuos puedan o no adquirir su estatus de ciudadanía en función de su contribución con el mercado laboral. Con esta nueva forma de entender el contrato social se corre el riesgo de convertir determinados derechos sociales en simples oportunidades de integración en el mercado laboral.

La centralidad en el empleo sería el elemento más característico de las políticas de activación. Frente a los problemas individuales y/o sociales que se presentan (exclusión social, falta de oportunidades, etc.), el camino a seguir es el marcado por el mercado de trabajo, la participación en él. Así, la categoría de ciudadanía se convierte en un problema de solución mercantil, sólo aquellos que participan en el mercado de trabajo tendrán las condiciones adecuadas para adquirirla, como la autora señala, "el trabajo se convierte en un prerrequisito de acceso a la ciudadanía" (Serrano 2007). Con este viraje de una ciudadanía de corte social a una centrada en la participación en el mercado, los parámetros de exigencia hacia el individuo han cambiado; las políticas de activación se centran en una disponibilidad total de éste con las exigencias del mercado. En este mismo punto, uno de los factores clave sería el paso de la seguridad, propia del Estado social protector, a la movilización. En su discurso, las instituciones europeas presentan como inevitables las actuales condiciones del mercado, son el precio a pagar para convertir la economía del continente en una de las economías más competitivas y eficaces del mundo (ver European Commission 2007).

La última característica de las políticas de activación hace referencia a cómo el Estado interviene en la construcción de un nuevo individuo el cual ha de dejar de ser pasivo para pasar a ser activo. En ese sentido se pretende el cambio de las actitudes y conductas individuales que permitan superar el estadio anterior de solidaridad vinculado a una ciudadanía que la UE da como ya superada. La solidaridad por parte del Estado, en línea con los dos rasgos anteriores, no permite el desarrollo óptimo de la economía la cual necesita una mano de obra que se adapte a sus cambiantes necesidades. Las políticas de activación buscarían dicho objetivo motivando a los individuos de tal forma que abandonen aquel estado de dependencia y desresponsabilización social (provocado por la solidaridad) para pasar a ser individuos responsables de su propio devenir laboral. 


\section{La popularización de la flexiguridad}

Cuando afirmamos que el concepto de flexiguridad es una nueva moda nos queremos referir a la proliferación de espacios reales y simbólicos que dicho concepto ha conquistado en los últimos años. Nominalmente hablando, el concepto de flexiguridad nace en 1995 en unos discursos realizados por el sociólogo holandés Hans Adriannsens sobre el nuevo panorama socioeconómico que, como ya comentamos en apartados anteriores, se caracterizaba por la consolidación de un capitalismo global y que supuso el replanteamiento de las relaciones entre empresario y trabajador en busca de un beneficio más a corto plazo para el primero condicionando la estabilidad laboral del segundo. Dicho concepto hacía referencia a la práctica política que estaba llevando a cabo el Ministerio holandés de Empleo y Asuntos Sociales combinando flexibilidad y seguridad en el empleo. Desde el punto de vista de Adriannsens la flexiguridad suponía un cambio de la seguridad laboral hacia la seguridad en el empleo al mismo tiempo que un cambio de actitud hacia la flexibilidad (entendida como facilidad de contratación y despido), así como la creación de un sistema de seguridad social flexible y activo (Wilthagen y Tros 2004). Dicho lo anterior, cabe resaltar que la novedad que supuso la adopción del término por aquel entonces no se vio correspondida con un cambio sustancial en la política de empleo de Holanda. En ese sentido, si bien el concepto se acuñó originariamente en este país, las políticas de flexiguridad se empezaron a observar, en mayor o menor medida, en otros Estados (Wilthagen 1998).

Desde aquella aproximación al concepto, su centralidad ha ido aumentando cada vez más hasta convertirse en uno de los pilares en los que se asienta el MSE. De hecho, el concepto se ha convertido en el último "fichaje" de la Comisión Europea para continuar en su línea de propulsar el conocimiento y las "buenas prácticas" (Keune \& Jepsen 2007). En la actualidad ocupa infinidad de espacios en todos los frentes en cuanto a políticas de empleo en la UE se refiere ${ }^{1}$. Estos espacios pueden englobarse en dos grupos: por un lado, los que están a la búsqueda de la legitimidad y la naturalización a través de la apelación al mundo científico, el cual ha dado cobertura al concepto en espacios no reservados para él (Serrano 2007). Son numerosas las colaboraciones entre la UE y científicos competentes en la materia a través de los grupos de trabajo, think tanks, seminarios, etc. Así, un amplio material teórico y empírico sobre el concepto se encuentra en un apartado específico en la página

${ }^{1}$ En ese sentido, España no se ha quedado atrás haciéndose eco del concepto de flexiguridad. Los principales medios de comunicación han recogido el debate que suscita dicho concepto, desde noticias que hablan del debate de los principales partidos sobre cómo enfocar el concepto en sus programas ideológicos hasta artículos de opinión expresando el apoyo o alejamiento a la flexiguridad, pasando por late night televisivos que le dedican el monólogo inicial del presentador, siempre de tipo humorístico e irónico, al concepto, un concepto, según sus palabras, "contradictorio". 
web de la Comisión Europea (European Employment Strategy, 2007). Por otro lado, la extensión de una literatura de corte científico que no busca justificar las políticas de la UE ha ayudado enormemente a una comprensión distinta del concepto, articulando y destapando características del concepto que, desde el punto de vista de los que buscan su legitimación, no parece existir.

\section{En busca de la legitimación}

Un análisis pormenorizado a los documentos sobre flexiguridad de la Comisión Europea permite encontrar evidencias de cómo la implementación del concepto de flexiguridad está en un estadio más avanzado de lo que lo está en el plano legitimatorio. Es decir, la forma en que se presenta en los documentos oficiales nos permite aproximarnos a la idea de una herramienta necesaria para el buen desarrollo del MSE, en ningún momento se nos presenta la flexiguridad como elemento que podría o no ayudar al logro de los objetivos propuestos en Lisboa. En ese sentido, el trabajo por parte de la Comisión de Empleo y Asuntos Sociales de la UE, con la colaboración de figuras del mundo científico ${ }^{2}$, parece haber conseguido que el concepto de flexiguridad haya pasado rápidamente del debate interno al hecho consumado. Flexiguridad, al igual que las políticas de activación, no se presenta como una posible vía de solución para conseguir que la economía europea sea la más fuerte y competitiva del mundo de cara al 2010, sino que se ha convertido en la condición sine qua non para ello.

La flexiguridad pretende garantizar que los ciudadanos de la UE puedan disfrutar de un elevado nivel de seguridad del empleo, es decir, que tengan la posibilidad de encontrar fácilmente un puesto de trabajo en todas las etapas de su vida activa y buenas perspectivas de desarrollar su carrera profesional en un entorno económico rápidamente cambiante. Asimismo, pretende ayudar tanto a los empleados como a los empleadores a aprovechar plenamente las oportunidades que ofrece la globalización. Así pues, se trata de crear un contexto en el que seguridad y flexibilidad puedan reforzarse mutuamente.

(Comisión Europea, Hacia los principios comunes de la flexiguridad: más y mejor empleo mediante la flexibilidad y la seguridad. pag. 4)

El aspecto cuantitativo es importante a la hora de querer justificar una idea. Con respecto al tema de la flexiguridad son muchos los pasos que se han dado en menos de dos años en busca de dicha justificación. Grupos de trabajo, encuentros, seminarios y conferencias sobre el concepto de flexiguridad se han ido desarrollando a lo largo del año 2006 y principios de 2007 hasta terminar con un documento oficial

\footnotetext{
${ }^{2}$ Economistas como Jochen Kluve (Director del departamento "Labor markets, Population, Health", RWI Essen) o John Hudson (Universidad de Bath) han sido referentes en la elaboración y definición del concepto de flexiguridad para la Unión Europea.
} 
sobre el concepto (European Comisión, 2007). Las dos características fundamentales de dichos pasos son la búsqueda de una concreción en el problema a tratar (la consecución de los objetivos de Lisboa) y los diagnósticos comunes apuntando al concepto de flexicurity como "vacuna" para todos los "males".

Flexiguridad desde esta perspectiva se entiende como la aproximación integral ideal para la consecución de los objetivos fijados en Lisboa a través de más y mejores empleos, al mismo tiempo que se moderniza el MSE. Los objetivos principales de esta aproximación son aumentar el nivel de seguridad en el empleo de los ciudadanos europeos y aumentar la facilidad de encontrar trabajo en los diferentes estadios de la vida laboral, manteniendo unas buenas posibilidades de desarrollo de la carrera profesional.

En cuanto a su definición, la Comisión Europea entiende el concepto como una estrategia integrada de fortalecimiento de la flexibilidad y la seguridad en el mercado de trabajo (Comisión Europea, 2007). Flexibilidad hace referencia, en primer lugar, a las transiciones durante la vida del empleado, pasando de la escuela al trabajo, de un trabajo a otro, de una situación de desempleo o inactividad a una situación de empleo, y del trabajo a la jubilación. Una transición laboral siempre en términos ascendentes. En segundo lugar, complementando lo anterior y, en nuestra opinión, más importante, la Comisión, hace referencia a los contratos indefinidos como los actores que no tienen por qué perder en esta nueva situación, cuestionando su carácter positivo con respecto a las transiciones.

Por un lado, la flexibilidad hace referencia a los avances logrados («transiciones») en el curso de la propia vida: del colegio al trabajo, de un empleo a otro, del desempleo o la inactividad al trabajo y del trabajo a la jubilación. No se limita a la mayor libertad de las empresas para contratar o despedir y no implica que los contratos por tiempo indefinido hayan quedado obsoletos. Alude a la progresión de los trabajadores hacia mejores empleos, a la «movilidad ascendente» y al desarrollo óptimo del talento.

(Comisión Europea, Hacia los principios comunes de la flexiguridad: más y mejor empleo mediante la flexibilidad y la seguridad. pag. 5)

Por otro lado, la Comisión Europea entiende que hay que proveer de seguridad a través de la adquisición de habilidades por parte de los trabajadores, dándoles apoyo y ayuda para que encuentren nuevos empleos, con adecuados beneficios de desempleo que faciliten su transición y ofreciéndoles oportunidades para su entrenamiento, especialmente a aquellos con menores habilidades o mayor edad. Así la seguridad deja de ser entendida como la certeza del trabajador para mantener un trabajo y, al

${ }^{3}$ Los principales objetivos del tratado eran: crear una Europa más democrática y transparente, situar a la UE en el mundo, ser un continente articulado en los derechos y los valores; ser un continente con más justicia, libertad y seguridad; y crear políticas que aumentasen la calidad de vida de sus ciudadanos. 
igual que en el caso de los contratos indefinidos, la seguridad se nos presenta como algo superado e inferior a la nueva propuesta.

Por seguridad, por otro lado, no sólo se alude a la seguridad de conservar el propio puesto de trabajo: se trata de equipar a las personas con las capacidades que les permitan progresar en su vida laboral y ayudarlas a encontrar un nuevo empleo. Pero también se trata de ofrecer unas prestaciones de desempleo adecuadas para facilitar las transiciones e incluye, por último, oportunidades de formación para todos los trabajadores, especialmente los poco cualificados y los de más edad. (Comisión Europea, Hacia los principios comunes de la flexiguridad: más y mejor empleo mediante la flexibilidad y la seguridad. pag. 5)

En nuestra opinión, el desarrollo del concepto nos muestra una balanza desequilibrada entre los dos conceptos a los que hace referencia: la flexibilidad y la seguridad. Esto se deriva de cómo la Comisión define ambos términos. Con flexibilidad, se hace referencia a un concepto claro, transiciones, que se traduce en movimientos laborales concretos (de la escuela a un trabajo, de un trabajo al desempleo, de la inactividad al trabajo...y así hasta la jubilación). Con seguridad, el plano semántico cambia, la idea clave es "ser garante" en los períodos de no trabajo, el Estado ha de ser el garante de la adquisición y renovación de habilidades por parte de la gente, ha de ser garante de adecuados beneficios al desempleo para facilitar aquellas transiciones $\mathrm{y}$, por último, ha de ser garante de que las oportunidades de entrenamiento serán para todos los trabajadores (European Commission 2007). Flexibilidad se convierte, sin duda, en el elemento que más peso tendría en la balanza, ya que la referencia a transiciones implica un hecho "visible" para el individuo que tendrá que asumir que las reglas del juego han cambiado. El individuo sabe que existirá un periodo en el que no trabajará. Seguridad, en cambio, se centra exclusivamente en dichos periodos de no trabajo. El Estado aportará "garantías" que permitan al individuo estar a punto para un futuro empleo pero sin garantizárselo. Así pues, con este tipo de seguridad al individuo se le asegura para "estar listo para el empleo" pero sin saber si tendrá un empleo mañana.

A la hora de destacar los componentes en los que deben incidir las políticas de flexiguridad, la Comisión Europea los engloba en torno a cuatro puntos:

- $\quad$ Acuerdos contractuales flexibles y fiables teniendo en cuenta dos perspectivas, la de los dos actores principales (empleador y empleado) y la de "los que están dentro" y "los que están fuera" del mercado laboral. Todo ello a través de leyes laborales, convenios colectivos y una organización adecuada para los trabajos modernos.

- Estrategias globales de aprendizaje permanente que garanticen la empleabilidad y adaptabilidad constante de los trabajadores, prestando especial atención a los trabajadores más vulnerables.

- Politicas activas del mercado laboral eficaces que ayuden a las personas a afrontar los cambios rápidos, disminuyan los tiempos 
de periodo de desempleo y faciliten las transiciones entre puestos de trabajo.

- Modernos sistemas de seguridad social. Que apoyen la renta de las personas de manera adecuada, que fomenten el empleo y facilite la movilidad en el mercado. En este punto también se hace mención de que los sistemas de seguridad social presten ayuda a las personas para que puedan conciliar su vida laboral y familiar.

La lógica bajo la cual descansan dichos componentes debiera encontrar en la Legislación de Protección de Empleo un gran impedimento para el progreso económico, pues aunque reduce el número de despidos, también disminuye en opinión de la comisión el número de desempleados que consiguen trabajo. Del mismo modo, dichos planteamientos de la Comisión, apoyándose en estudios empíricos, afirman que los individuos se sienten más protegidos cuando reciben beneficios por desempleo que con políticas estrictas de protección contra los despidos.

"Este es el caso, en particular, de toda legislación estricta de protección del empleo contra los despidos por razones económicas. De acuerdo con datos obtenidos a partir de análisis, una legislación estricta de protección del empleo reduce el número de despidos, pero también las posibilidades de reinserción en el mercado laboral tras periodos de desempleo."

(Comisión Europea, Hacia los principios comunes de la flexiguridad: más y mejor empleo mediante la flexibilidad y la seguridad. pag. 5)

A través del benchmarking y del Modelo Abierto de Coordinación, buscando medir cuantitativamente las evoluciones de los diversos países miembros para la consecución de los objetivos (Serrano 2007, Keune \& Jepsen 2007), la Comisión Europea ha redactado una serie de principios comunes que ha de tener el concepto de flexiguridad para poder crear códigos comunes con los que codificar y, posteriormente, comparar países. Dichos principios se basan, según la comisión, en la experiencia y la evidencia analítica, es decir, se generalizan unos principios a escala continental a partir de las evidencias demostradas en determinados países. De nuevo encontramos evidencias de la pretensión por parte de la UE de crear un proyecto político común utilizando como modelo los resultados estadísticos de países concretos.

Entrando en mayor detalle al análisis de los cuatro principios antes descritos podemos hacer dos tipos de comentarios, uno de corte cuantitativo y el otro de corte cualitativo, los dos nos ayudarán a corroborar una de nuestras hipótesis de partida acerca de la carga desigual en el concepto de flexiguridad. Con respecto al análisis cuantitativo, de los cuatro componentes del concepto de flexiguridad, tres hacen referencia a la dimensión de la flexibilidad ("Acuerdos contractuales flexibles y fiables", "Estrategias globales de aprendizaje permanente" y "Políticas activas del mercado laboral eficaces") mientras que sólo uno lo podríamos situar en la dimensión de la seguridad ("modernos sistemas de seguridad social"). 
En el plano cualitativo, la evidencia de un concepto de flexiguridad no balanceado (Keune \& Jepsen 2007) se hace mucho más evidente. El contenido principal de los cuatro componentes del concepto tiene un mismo inspirador común: la flexibilidad del mercado de trabajo a través de la adaptabilidad del trabajador a las diferentes situaciones que le van a surgir, en ese sentido el Estado ha de ser el garante de que los periodos de inactividad laboral sean los más cortos posibles. En relación al primer componente, la Comisión hace referencia a que los acuerdos han de ser tanto fiables como flexibles, para ello parece imprescindible "modernizar" leyes, convenios y acuerdos de antaño que se adapten al entorno cambiante y las demandas del "mercado".

El segundo, aprendizaje a lo largo de toda la vida, se especifica como la estrategia para asegurar la adaptabilidad y empleabilidad del trabajador, es decir, asegurar que el trabajador sabrá llevar la etapa de flexibilidad por la que le tocará pasar.

Las políticas efectivas de mercado laboral activo son otro caso evidente de referencia a la flexibilidad. En este caso podríamos interpretar dichas políticas como una especie de políticas de "resocialización" del individuo en el nuevo panorama laboral caracterizado por los cambios y las transiciones.

El último componente, si bien hemos indicado anteriormente su referencia directa al plano de la seguridad, no esconde uno de sus objetivos primordiales: facilitar la movilidad del mercado laboral, es decir, lo que en última estancia significa fomentar una mano de obra que se adapte a las "inclemencias" que puedan surgir, es decir, dar por sentado que el trabajador tendrá a lo largo de su vida diferentes trabajos.

Así, a partir de la definición y principios comunes en los que han de basarse las políticas de flexiguridad se plantean diferentes caminos para implementar dicha filosofía en función del país miembro. Cada país miembro tendrá así una especie de receta específica para implementar la flexiguridad en función de su situación previa y a partir de aquella definición y principios comunes. En ese sentido, la Comisión Europea trabaja de nuevo en la creación de una solución común, la flexiguridad, que variará en función de los "síntomas previos" de cada país. Dicha solución, que no deja de ser un modo de regulación, pretende socavar los cimientos de la cuestión social tal como se entendía hasta el momento en busca de instaurar un nuevo contrato moral que enfatiza el autogobierno del sujeto (Walters y Haahr, 2005; Serrano 2007), como centro de todos los derechos, pero también de todas las obligaciones. Ahora bien, mientras los derechos están sufriendo un proceso de contracción con las obligaciones, el Estado ha pasado la pelota ("passed the buck") a los individuos.

Dicho de otro modo, se deja al albur de los gobiernos de los Estados miembros cómo se desarrollará el marco institucional que dé seguridad a los trabajadores en proceso de transición. No se concreta, por ejemplo, una exigencia mínima en términos de subsidios de desempleo, sistema de pensiones, formación para el empleo, etc. La concepción de Schmid (1995) de los mercados transicionales de trabajo aportaría, en este sentido, una especificación, y un compromiso, mucho mayor, que, no exenta de dificultades, plantea la necesidad de arreglos institucionales que protejan de los riesgos en las transiciones individuales. 


\section{Buscando la comprensión}

Paralelamente al proceso de legitimación del concepto de flexiguridad que anteriormente hemos descrito, es necesario encontrar un camino que nos ayude a comprender el concepto a través de posiciones que cuestionan y desmenuzan el mismo. El punto de partida de esta sección serán los trabajos que desde diferentes perspectivas problematizan el concepto de la flexiguridad y se enfrentan al planteamiento que de él hace la UE.

Desde nuestro punto de vista, flexiguridad nace en una época de fascinación por la actividad empresarial y el nuevo empoderamiento del mercado, los cuales han provocado una individualización de las relaciones sociales y una gradual disminución de los acuerdos colectivos de antaño (Nanteuil-Miribel \& Nachi 2004). Esto no quiere decir que el concepto haya nacido con el signo de ir en contra de los intereses del trabajador, por el contrario, todo concepto parte de un significado abierto en buena medida y son sólo los agentes que lo utilizan quienes lo revisten de determinadas cargas significantes.

Un instrumento que ayude a mantener la protección social frente a la fluctuación, inestabilidad y la fragmentación crecientes sería necesario y, en ese sentido, flexiguridad podría ser el adecuado, al menos tomando en cuenta los significados a los que hace referencia. La realidad, en cambio, muestra la flexiguridad como una herramienta estratégica al servicio de las características del mercado, un mercado que fomenta cada vez más una mano de obra adaptable a la infinidad de situaciones. Además, la UE, en su función de agente entre los intereses económicos y los políticos (Keune \& Jepsen, 2007) ha encontrado en dicho elemento la clave del éxito para la consecución de los objetivos anteriormente comentados. La filosofía del MSE no permite acotar o delimitar la flexiguridad, lo que provoca, irremediablemente, la proliferación de escenarios diversos y, consecuentemente, la corrupción de su significado.

En ese sentido, los Estados se presentan ante la sociedad con las "manos atadas" con respecto al mundo del trabajo, pues éste se encuentra inexorablemente relacionado con el mercado. Son numerosos los autores que promulgan de una u otra manera la intervención del Estado. Dicha institución, lejos de desaparecer, debe tener la tarea de reconocer, apoyar y legitimar las regulaciones que se den en el mundo del trabajo para así poder entender de una forma nítida la flexiguridad y, de esa manera, llegar a saber a ciencia cierta cuáles son las ventajas y los inconvenientes de aplicar políticas que afectan a sus ciudadanos. Desde una perspectiva económica, Gautié (2005) introduce un elemento apenas tratado en los documentos analizados de las instituciones europeas: la protección del empleo. Gautié cuestiona la afirmación de los economistas europeos que consideran dicha protección como una barrera para el crecimiento de empleo. El autor afirma que, utilizando un esquema meramente fordista, el término flexiguridad hace referencia a dos conceptos antagónicos. En ese sentido, es la protección del empleo el instrumento ideal para limitar la flexibilidad y asegurar la seguridad. Por otro lado, desde una perspectiva más sociocultural, Nanteuil-Miribel y Nachi (2004) se refieren a la posibilidad de crear una jerarquia de valores que permita combinar las prácticas del mercado con 
elementos que no se pueden reducir al mismo. Así, el Estado ha de tomar partido e intervenir en temas nucleares como el origen de la inseguridad laboral, imponiendo multas por las prácticas abusivas, reaccionando frente a los nuevos riesgos sociales, etcétera. En definitiva un Estado capaz de intervenir frente a la variedad de situaciones que pueden surgir como consecuencia de los numerosos cambios de una sociedad.

\section{La institucionalización de la individualización: la flexiguridad}

Una de las características más reconocidas por diferentes autores recientes acerca del individuo actual es su obligada flexibilidad, tanto en el plano laboral como en el personal (Sennett 2000). Dicha flexibilización hace referencia a la constante adaptación del individuo a los vaivenes que le plantea la sociedad, en este caso el paso de una relación de cierta estabilidad con el trabajo a una de constante cambio. Esto implica la materialización de un nuevo sujeto que estaría acorde con la pretendida identidad común europea anteriormente comentada. En esta nueva etapa, parecen quedar caducados signos anteriores de protección laboral, como el convenio colectivo, el Estado interventor o, incluso, la identidad o cultura obreras. En este sentido, el desarrollo de la flexiguridad no otorga ningún papel a la negociación colectiva ni a los sindicatos en general, tan importantes en el desarrollo del MSE, pero que desaparece cuando se trata la cuestión de la flexibilidad de los mercados.

Lo que se pretende es modelar al individuo para que sea juez y parte de su futuro laboral, un individuo que encuentre dentro de sí los recursos, caminos y respuestas a la hora de afrontar su futuro laboral. Dicha normalización de la individualización encontraría su reflejo en los discursos organizacionales, los cuales, en nuestra opinión, están conectados directamente con las políticas de empleo de la UE.

En busca de ganar la batalla en el terreno del lenguaje, la UE recoge el concepto de flexiguridad del modelo holandés, y posteriormente del alemán y del danés, sin escudriñar al máximo la aplicación y el contexto en el cual se desarrolla en dichos países. De hecho, lo que realmente se aplicó en aquellos países no fue lo que hoy en día conocemos como políticas de flexiguridad, sino más bien se tomaron una serie de reformas en pos de flexibilizar el mercado laboral (Wilthagen 1998, Wilthagen $\&$ Tros 2004). Interesadamente, flexiguridad se convierte en la estrella principal del Tratado de Lisboa del año 2000 aunque realmente no se explicitara en ningún momento del documento la palabra flexiguridad (Keune \& Jepsen 2007). Fue posteriormente, en la revisión de dicho tratado donde, de repente, aparece el concepto en una especie de carrera legitimatoria de una idea que años antes apenas aparecía en los documentos de la Comisión. Siete años después, tras numerosos encuentros, conferencias, seminarios, etc., la Comisión Europea publica un documento con los principios comunes de la flexiguridad, así como el "state of the art" en los diferentes países miembros.

Anteriormente, hemos comentado las características fundamentales de las políticas de activación dentro de la EEE. Sus principios fundamentales tendrían como 
raíz central al individuo, el cual ha pasado de tener estatus de ciudadanía a tener que ganarse dicho estatus a través de su activación y movilización (Serrano 2007). El trasfondo de dicho viraje es la desresponsabilización del Estado y la imposición de más deberes sobre el individuo.

Con el concepto de flexiguridad hallamos un proceso similar: a la hora de encontrar trabajo, el individuo se ha convertido en el único responsable de encontrarlo o no. Si bien los textos de la Comisión Europea refuerzan la idea de que tanto las empresas como los individuos saldrán reforzados de la aplicación de dichas políticas, su discurso muestra cierta complacencia con respecto a favorecer a las primeras debido al nuevo escenario económico surgido.

"Para poder cumplir los objetivos de Lisboa y lograr asi más y mejor empleo, los ciudadanos, las empresas, los Estados miembros y la propia Unión precisan de nuevas formas de flexibilidad y seguridad. Los ciudadanos necesitan cada vez más la seguridad del empleo, y no la del puesto de trabajo, ya que cada vez son menos los que conservan el mismo puesto de trabajo de por vida. Las empresas, y sobre todo las PYME, deben ser capaces de adaptar su mano de obra a unas condiciones económicas cambiantes. Han de poder contratar a trabajadores que dispongan de una mejor combinación de capacidades y que sean más productivos y adaptables, aumentando así su capacidad de innovación y su competitividad."

(Comisión Europea, Hacia los principios comunes de la flexiguridad: más y mejor empleo mediante la flexibilidad y la seguridad. pag. 3)

Mientras que al individuo no se le pregunta o se le pregunta de forma sesgada (European Employment and Social Policy, 2006) sobre cómo desearía que fuese el trabajo, la UE recibe con brazos abiertos las noticias sintomáticas que transmiten las empresas sobre el mercado: hay que flexibilizar para poder hacer frente a los retos y las oportunidades que nos presenta la globalización. Por otro lado, lo que conocemos y entendemos por seguridad ha sufrido una metamorfosis. La seguridad en el texto analizado se convierte en un elemento que trata de tapar los agujeros que deja la flexibilidad. En términos laborales ya no se puede entender ese concepto como el que aseguraba al trabajador la consecución o el mantenimiento del puesto de trabajo. Y además se legitima en el apoyo que estos planteamientos reciben en las encuestas de opinión realizadas a ciudadanos europeos.

"Los ciudadanos europeos aceptan la necesidad de adaptación y cambio. De ellos, un $76 \%$ admite que los empleos de por vida con un mismo empleador son cosa del pasado y considera que, hoy en día, ser capaz de cambiar de empleo fácilmente es una baza útil para encontrar trabajo; un $72 \%$ afirma que se deberían flexibilizar los contratos de trabajo para fomentar la creación de empleo; y, por último, un $88 \%$ ha indicado que la formación regular mejora las posibilidades de carrera. La respuesta estratégica global de la UE a los desafios y las oportunidades de la globalización se encuentra en la Estrategia de Lisboa renovada para el Crecimiento y el Empleo. El Consejo Europeo invitó a los Estados miembros a «que en los programas nacionales de reforma se elaboren de manera más sistemática estrate- 
Francisco J. Tovar y Juan Carlos Revilla La institucionalización de la individualización del trabajo...

gias detalladas de actuación para mejorar la adaptabilidad de los trabajadores y las empresas»."

(Comisión Europea, Hacia los principios comunes de la flexiguridad: más y mejor empleo mediante la flexibilidad y la seguridad. pag. 3 y 4)

El sentido que se pretende dar al concepto de flexiguridad en el citado documento tiene mucho que ver con la psicologización del trabajo, pues trata de naturalizar la responsabilidad de la situación al individuo, ya sea trabajador como desempleado, si bien la Comisión considera al primero el culpable de la situación del segundo, pues considera que los 'insiders', con su sabida buena protección de empleo, son los responsables de que los que buscan trabajo, los 'outsiders', sólo consigan trabajos precarios o no sean capaces de conseguir empleo durante largo tiempo. Los 'insiders', según la Comisión, estarían así poniendo en riesgo la sostenibilidad del sistema de protección social y, consecuentemente, la cohesión social de Europa.

"De acuerdo con datos obtenidos a partir de análisis, una legislación estricta de protección del empleo reduce el número de despidos, pero también las posibilidades de reinserción en el mercado laboral tras periodos de desempleo. Al decidir la conveniencia o no de contratar a nuevos trabajadores, las empresas tienen en cuenta la probabilidad de incurrir en altos costes por despido en el futuro. Esto es especialmente pertinente en el caso de las pequeñas empresas. Por otra parte, los análisis indican que, aunque las repercusiones de una política estricta de protección del empleo en el desempleo total son limitadas, ésta puede perjudicar a aquellos grupos que tienen más posibilidades de encontrar obstáculos a la hora de acceder al mercado laboral, como los jóvenes, las mujeres, los trabajadores de más edad y los desempleados de larga duración."

(Comisión Europea, Hacia los principios comunes de la flexiguridad: más y mejor empleo mediante la flexibilidad y la seguridad. pag. 6)

Otra forma de psicologización del trabajo aparece en relación al discurso sobre la formación y el entrenamiento a lo largo de la vida. Tales ejercicios, enfocados principalmente para aquellos en situación de desempleo, han de tener, en opinión de la Comisión, un cierto coste para los individuos que la "disfruten" a pesar de que, en última instancia, incidan en la productividad de las empresas en las que trabajaran a posteriori.

"Sin embargo, con demasiada frecuencia los únicos que se benefician de estas inversiones son los trabajadores altamente cualificados. Aquellos para los que la necesidad de aprender es acuciante, como los trabajadores poco cualificados, los trabajadores con contratos temporales, los autónomos y los de más edad, son los más afectados por la falta de inversión en formación. Uno de los factores que puede disuadir a las empresas de invertir en el desarrollo de las capacidades es el temor a que, una vez formados, sus empleados puedan ser contratados por otros empleadores. El reparto de los costes, por ejemplo mediante la asignación de fondos a escala sectorial, puede evitar parcialmente este problema." 
(Comisión Europea, Hacia los principios comunes de la flexiguridad: más y mejor empleo mediante la flexibilidad y la seguridad. pag.7)

Con referencia a la seguridad, uno de los puntos centrales, como ya se ha comentado anteriormente, es el concepto de empleabilidad el cual se entiende como la habilidad del trabajador para encontrar empleo en un momento dado. Dicho elemento ha sido altamente potenciado por la Comisión Europea desde el año 2000. Waltery \& Vielle (2004) analizan cómo la empleabilidad vendría altamente condicionada por lo que denominan "la naturaleza temporal de la seguridad". Según estos autores se pueden distinguir dos tipos de seguridad en la empleabilidad en función del tiempo: uno a corto plazo que remitiría a la responsabilización directa del individuo a través de su propia habilidad para encontrar trabajos. Otro, que haría referencia a la seguridad a largo plazo y distribuiría la responsabilidad entre el trabajador y las políticas de empleo, esta seguridad haría referencia al desarrollo (entrenamiento y perfeccionamiento) de habilidades por parte del individuo y, también, por parte de las empresas ofertando empleos adecuados a dichas habilidades. Así, aparecen elementos paradójicos relacionados con la seguridad en el concepto de empleabilidad en dos vertientes, a corto y largo plazo. A corto plazo la seguridad se convierte en responsabilidad a la hora de encontrar trabajo. El individuo es el problema y la solución a la hora de encontrar trabajo, teniendo valor ese dicho perteneciente al lenguaje popular de "si no encuentra trabajo es porque no quiere". En ese sentido, parece que la dirección de la política europea de flexiguridad es generar la mayor tasa de empleo a cualquier coste, debiendo el individuo aceptar empleos de baja calidad antes que estar en desempleo. A largo plazo la seguridad es entendida como la creación de una infraestructura suficiente para el desarrollo de habilidades que no son necesariamente aprovechables a corto plazo. Dicho desarrollo, de nuevo, no asegura la reinserción futura del trabajador al mercado laboral, quizás aumenta la probabilidad, pero nunca la asegura.

Como venimos observando es la nueva definición de seguridad, muy amplia y cargada de ambigüedades, la que hace posible la individualización del trabajo. Una seguridad que ha perdido la fuerza que le caracterizaba en épocas anteriores a través de los estatutos laborales, los convenios colectivos, la protección social, etc., para convertirse en la pareja ideal de la flexibilidad.

Si cambiamos la óptica de las instituciones europeas por una óptica más micro, centrada en el mundo gerencial, se pueden encontrar conexiones espacio-temporales entre las pretensiones de unas y otras. Por un lado, la tendencia hacia la individualización de las relaciones laborales la podemos encontrar en una primera etapa en las corrientes gerenciales a finales de $\operatorname{los} 80 \mathrm{y}$, sobre todo, principio de los 90 . La Comisión Europea, desde mediados de los noventa y en una segunda etapa, recoge su envite y reconoce dicha necesidad a través de su discurso sobre el mercado flexible y la necesidad de una mano de obra adecuada al mismo (European Comisión 2007).

Existen numerosas evidencias para demostrar cómo la vertiente doctrinal de la Dirección de Empresas se ha encaminado en los últimos quince años hacia una 
psicologización-individualización de las relaciones entre el trabajador y la organización. Crespo et al. (2005) realizan un recorrido por la evolución de las formas de sujeción del individuo desde inicios del siglo XX hasta los manuales gerenciales de los noventa evidenciando cómo se ha pasado de unas formas de sujeción coercitivas $\mathrm{y}$, posteriormente, humanizadas con la característica de ser comunes a todos los trabajadores, a otras formas de sujeción de corte moral donde cada individuo ha de interiorizar la cultura organizacional de la empresa a la que pertenece.

La sujeción moral o autosujeción busca la participación del individuo en la cultura organizacional hasta el punto de apropiarse e interiorizar su contenido. Dicha participación acaba provocando una identificación del individuo con la empresa de tal forma que se produce una modificación de los valores personales para que coincidan con los de la organización (Lawler 1994), dicha modificación puede terminar incluso con la sustitución de los primeros por los segundos en el individuo.

Uno de los modos de gestión empresariales que más está fomentando la autosujeción del individuo es la gestión por competencias. Las competencias del individuo están compuestas tanto por sus habilidades o conocimientos teóricos para desarrollar la tarea como también por aquellas denominadas soft skills como la actitud, la empatía, etc., es decir las características más psicológicas del individuo (Tovar y Revilla 2009).

En ese sentido, la responsabilidad del mantenimiento o pérdida del empleo recae en la puesta en práctica de los elementos más personales del individuo mientras que, al mismo tiempo, desaparece la responsabilidad organizacional y, en última estancia, la responsabilidad estatal.

Así pues, se ha instalado una autosujeción que invade claramente la subjetividad de los individuos que trabajan en la organización a través de procesos doctrinales de corte económico e ideológico. La organización se intromete en el individuo, pidiéndole una involucración global (valores, actitudes, motivaciones, proyectos vitales) en pos del funcionamiento ideal de la organización (Crespo et al. 2005).

\section{Conclusiones}

A lo largo del presente artículo hemos ido abordando diferentes cuestiones que giran en torno al concepto de flexiguridad. No podemos entender el concepto de flexiguridad sin analizar antes lo que constituye y significa el MSE. Un modelo que se sigue construyendo sobre la marcha y en el que la multiplicidad de situaciones nacionales tiene cabida. Posteriormente serán las diferentes evaluaciones las que medirán cuantitativamente las evoluciones de los países y sus distanciamientos de la media europea.

Del mismo modo, hemos analizado las políticas de activación como el otro pilar importante en políticas de empleo. El exhaustivo análisis de Serrano (2007), comentado aquí someramente, permite indagar en la naturaleza polisémica y contradictoria de dichas políticas, situación paralela a la que sufre el concepto de flexiguridad. 
La evolución de los modos de sujeción promulgados por las necesidades del mercado nos muestra la tendencia hacia la individualización del trabajo que hemos planteado como hipótesis de partida. Dicha individualización del lado empresarial aparece vinculada con la responsabilización total del individuo de su situación laboral. Así, es, en nuestra opinión, el concepto de flexiguridad un claro dinamizador de dicha responsabilización que no es más que la fase previa a procesos de individualización en las relaciones laborales.

Lo preocupante de la situación analizada es, como hemos comentado en algún momento, la tendencia a la no problematización de la nueva situación y, del mismo modo, a naturalizar de una manera tan concreta el concepto de flexiguridad como el ideal para alcanzar los objetivos propuestos en el Tratado de Lisboa. Sin querer entrar a cuestionar la idoneidad de dichos objetivos, es difícil encontrar formas alternativas para su consecución cuando las instituciones europeas ponen en funcionamiento toda su maquinaria de creación de discursos en busca de su autolegitimación.

En el proceso de no problematización, el discurso de la Comisión Europea busca instaurar una nueva fase a través del concepto de flexiguridad, acorde con las exigencias de flexibilidad del mercado. En esa pretensión, cabe destacar cómo el concepto de seguridad está siendo constantemente dinamitado. Si bien encontramos la palabra inserta en el concepto de flexiguridad, la seguridad es la piedra que se quiebra a través de su discurso. En ese sentido, del análisis realizado en puntos anteriores podemos concluir cómo la seguridad, tal y como se entendía hasta hace bien poco, se ha convertido en algo obsoleto, antiguo. La Comisión Europea realiza todo un ejercicio de desapropiación del concepto de seguridad para definirla a partir del contexto de flexibilización. Así, flexibilización, concepto naturalizado y no problemático, es considerado por la Comisión como la piedra filosofal a partir de la cual construir todo un sistema de significantes dentro del cual estaría la (nueva) seguridad. La pretensión de naturalizar el nuevo significado del concepto de seguridad implica despojar al trabajador del elemento histórico más importante que le ha venido acompañando en su constante pulso con el empleador, que no es otro que la capacidad para establecer dinámicas colectivas de protección social, así como de defensa o mejora de las condiciones laborales y del empleo ante el Estado y ante la empresa. En ese sentido, las movilizaciones que recientemente cuestionan la introducción de las políticas de flexiguridad en diferentes países europeos (Francia, Portugal e Italia) deben servir de carburante para seguir poniendo en cuestión la naturaleza benévola y no problematizada de determinadas políticas de empleo.

Consecuentemente consideramos que no es sólo necesario seguir trabajando en el análisis del concepto de flexiguridad de tal forma que sea más equilibrado y preciso, como afirman diferentes informes de partidos políticos europeos, sino que es pertinente recuperar el significado claro y uniforme de numerosos conceptos que están siendo modificados y/o erosionados con vistas a la consolidación de un determinado Modelo Social Europeo. 
Francisco J. Tovar y Juan Carlos Revilla La institucionalización de la individualización del trabajo...

\section{Bibliografía}

Alonso, L.E. (1999). Trabajo y ciudadanía. Estudios sobre la crisis de la sociedad salarial, Madrid: Trotta (2001). Trabajo y posmodernidad: el empleo débil. Madrid: Fundamentos. (2007). La crisis de la ciudadanía laboral. Barcelona: Anthropos.

Barbier, J.C. (2004). "Activation policies: a comparative perspective" en Serrano Pascual, A. (Ed.). Are activation policies converging in Europe? The European Employment Strategy for young people. Bruselas: ETUI.

Bauman, Z. (2000). Trabajo, consumismo y nuevos pobres. Barcelona: Gedisa. (2006) Modernidad líquida. Buenos Aires: Fondo de Cultura Económica.

Castel, R. y Haroche, C. (2001). Propriété privée, propriété sociale, propiété de soi. Paris: Fayard.

Castells, M. (2001). La sociedad Red. En La era de la información: economía, sociedad y cultura. Vol.1. Madrid: Alianza Editorial.

Comisión Europea (2007). Hacia los principios comunes de la flexiguridad: más y mejor empleo mediante la flexibilidad y la seguridad. Bruselas.

Commis. Europeenne (2003). L'emploi, l'emploi, l'emploi. Brussels: Task -force pour l'emploi.

Crespo, E.; Revilla, J.C. y Serrano, A. (2005). «La psychologisation politique du travail ». En A.Dorna (ed.) Psychologie Politique. Paris: L'Harmattan.

Crespo, E. \& Serrano, A (2002). «El discurso de la Unión Europea sobre la sociedad del conocimiento». Reis: Revista española de investigaciones sociológicas, $\mathrm{N}^{\mathrm{o}}$ 97, 2002, pags. 189-210.

European Commission (1994). White paper - European social policy: A way forward for the Union, Luxembourg: Office for Official Publications of the European Commission.

European Employment Strategy (2007). Flexicurity: the response to globalisation and demographics by combining flexibility and security http://ec.europa.eu/employment_social/employment_strategy/flex_meaning_en.h tm [Search: $7^{\text {th }}$ November 2007].

European Trade Union Confederation (2005). What is the "European Social Model" or "Social Europe"? http://www.etuc.org/a/111 [Search: 7th November 2007]. 
Francisco J. Tovar y Juan Carlos Revilla La institucionalización de la individualización del trabajo...

Fairclough, N. (2000). New labour, New language? Londres y Nueva York: Routledge.

Goetschy, J (1999). «The European Employment Strategy: Genesis and Development» en European Journal of Industrial Relations, Vol. 5(2), 117-137.

Handler, J. (2006). "Activation policies and the European Social Model" in M. Jepsen y A. Serrano (ed.) Unwrapping the European Social Model. Bristol: Policy Press.

Jepsen, M. y Serrano, A. (2006). Unwrapping the European Social Model. Bristol: Policy Press.

Keune, M. y Jepsen, M. (2007). "Not balanced and hardly new: the European Commision's quest for flexicurity" en Jørgensen, H. \& Kongshøj Madsen, P. (eds.): Flexicurity and Beyond, Copenhagen: DJØF Publishing.

Lawler, E. E. (1994). «From Job-Based to Competence-Based Organizations» en Journal of Organizational Behavior, Vol. 15, $\mathrm{n}^{\mathrm{o}}$ 1: 3-15.

Marshall, T.H. (1997). "Ciudadanía y clase social" en Revista Española de Investigaciones Sociológicas, ${ }^{\circ}$ 79, Julio-Septiembre.

Mertens, L. (1996). Competencia laboral: sistemas, surgimiento y modelos. Montevideo. Cinterfor/OIT.

Nanteuil-Miribel, M. y Nachi, M. (2004). «Flexibility and Security: what forms of political regulation? », TRANSFER, 10 (2), 300-318.

Rifkin, J. (1997). El fin del trabajo: Nuevas tecnologías contra puestos de trabajo: el nacimiento de una nueva era. Barcelona: Paidós.

Schmid, G. (1995). "Is Full Employment Still Possible? Transitional Labour Markets as a New Strategy of Labour Market Policy", Economic and Industrial Democracy, 16, 429-56.

Sennett, R (2000). La corrosión del carácter. Las consecuencias personales del trabajo en el nuevo capitalismo. Barcelona: Anagrama. (2006). La cultura del nuevo capitalismo. Barcelona: Anagrama

Serrano, A. (2009). «Regulación supranacional y despolitización del trabajo: el caso del paradigma de la activación», en E. Crespo, C. Prieto y A. Serrano Trabajo, subjetividad y ciudadanía: paradojas del empleo en una sociedad en transformación. Madrid: Editorial Complutense- CIS. 
Tovar, F.J. y Revilla, J.C. (2009). "An Alternative view of Competence-based Management " en Koistinen, P., Mósesdóttir, L. y Serrano Pascual A. (eds.) Emerging Systems of Work and Welfare. Brussels: Peter Lang.

Vielle, P. y Walthery, P. (2003). Flexibility and social protection. European Foundation for the Improvement of Living and Working Conditions, Dublin.

Walters, W. y Haahr, J. H. (2005). Governing Europe. Discourse, governmentality and European Integration. Nueva York: Routledge. Advances in European Politics.

Walthery, P. y Vielle, P. (2004). «Reconciling security with flexibility: a few questions», TRANSFER, 10 (2), 264-281.

Wilthagen, T. y Tros, F (2004). «The concept of "flexicurity": A new approach to regulating employment and labour market», TRANSFER, 10 (2), 166-186.

Wilthagen, T. (1998). «Flexicurity: A new paradigm for Labour Market Policy Reform? », Berlin: WZB Discussion Paper FS I, 184-242. 